\title{
AUS DER PRAXIS
}

\section{Die Ost-West-Tarifangleichung in der Metall- und Elektroindustrie}

\section{UNVORBEREITET IN DEN KAPITALISMUS}

Als am 1. Juli 1990 die D-Mark in der zu diesem Zeitpunkt noch bestehenden DDR eingeführt wurde, waren die Tarifvertragsparteien gefordert, die Einkommens- und Arbeitsbedingungen der Beschäftigten neu zu ordnen. Grundlage war der am 18. Mai 1990 von den Regierungen der Bundesrepublik Deutschland und der DDR geschlossene Vertrag zur Schaffung einer Währungs-, Wirtschafts- und Sozialunion. Beim Wechsel zur D-Mark wurde die bisherige Ost-Mark im Verhältnis 1:1 umgetauscht. Mit Einführung der D-Mark wurden in der DDR ökonomische Rahmenbedingungen hergestellt, auf die die dortigen Betriebe nicht vorbereitet waren. Die Vereinigung der beiden deutschen Staaten wurde mit dem Einigungsvertrag, vom 31. August 1990, am 3. Oktober 1990 wirksam. Die Lösung der ökonomischen Probleme hat die Regierung Kohl weitgehend auf die Tarifvertragsparteien und die Sozialversicherungsträger abgewälzt.

Das Gesetz über die Herstellung der Wirtschafts- und Währungsunion sowie der Einigungsvertrag schufen die rechtlichen Rahmenbedingungen für die Einführung des kapitalistischen Wirtschaftssystems. Die Betriebe der DDR mussten von einem auf den anderen Tag mit wesentlich produktiveren Betrieben vor allem aus West-Deutschland konkurrieren. In einem ersten Schritt sicherte die Treuhandanstalt den Fortbestand der Betriebe. Sie erhielten mit Einführung des D-Mark-Bilanzierungsgesetzes die Möglichkeit, Ausgleichsforderungen gegenüber der Treuhandanstalt zu bilanzieren, wenn die Eröffnungsbilanz andernfalls zu einer Überschuldung und damit zur sofortigen Insolvenz geführt hätte.

Mit Einführung der D-Mark musste für die Beschäftigten der DDR aber auch die Einkommenssituation neu geregelt wer- den. Das Gesetz zur Herstellung der Wirtschafts-, Währungs- und Sozialunion sah lediglich die Umstellung der Währung im Verhältnis 1:1 vor. Dieser politisch gesetzte Umrechnungskurs führte jedoch nicht zu einem ökonomisch begründbaren und sozial verträglichen Einkommensniveau, weil mit Herstellung der Wirtschafts- und Währungsunion bisher subventionierte Preise für die Güter des täglichen Bedarfs auf West-Niveau angehoben wurden. Mieten und öffentlich administrierte Preise stiegen erst schrittweise im weiteren Verlauf der ökonomischen Angleichung. Die Gewerkschaften und die Arbeitgeberverbände waren herausgefordert, die Arbeitsbedingungen der Beschäftigten unter den Prämissen der westdeutschen kapitalistischen Wirtschaftsordnung neu zu gestalten. Dies setzte zunächst einmal die Einrichtung einer entsprechenden Verbandsstruktur voraus.

\section{UNBELASTET IN DEN TARIF- POLITISCHEN NEUBEGINN}

Das Rechtssystem der DDR kannte zwar Tarifverträge, jedoch wurden sie nicht unter den Bedingungen einer Marktwirtschaft zwischen Tarifvertragsparteien mit unterschiedlichen Interessen verhandelt. Für die IG Metall stellte sich die Frage, welche Gewerkschaftsstrukturen es künftig in Deutschland geben sollte. Bereits am 6. Dezember 1989 vereinbarten die Spitzen der IG Metall der Bundesrepublik Deutschland und der IG Metall der DDR eine Grundsatzvereinbarung über den Gewerkschaftsaufbau. Am 6. Oktober 1990 beschloss die IG Metall der DDR ihre Auflösung zum 31. Dezember 1990. Die IG Metall für die Bundesrepublik Deutschland änderte ihre Satzung und erweiterte ihre Zuständigkeit auf Gesamtdeutschland. Diese Erweiterung bot die Chance eines politisch unbelasteten Neubeginns der Gewerkschaftsarbeit in den Betrieben der fünf neuen Länder. Die IG Metall hat deshalb auch nicht einfach die Mitglieder übernommen. Das politische Mandat, für die Beschäftigten zu sprechen und sie in Tarifverhandlungen zu vertreten, hat sie sich durch deren Beitritte erworben. Die IG Metall der DDR hatte ihren Mitgliedern den Übertritt empfohlen. Da zwischen der Herstellung der Wirtschaftsund Währungsunion und der Herstellung der deutschen Einheit eine Übergangszeit lag, in der noch das Recht der DDR galt, wurden die ersten Tarifverhandlungen noch von der IG Metall der DDR geführt unter Beteiligung und Begleitung von Vertretern der IG Metall aus der Bundesrepublik.

Spiegelbildlich entwickelte sich die Arbeitgeberseite. Nachdem der politische Rahmen für den Weg zur deutschen Einheit geklärt war, gründeten sich in der ehemaligen DDR Unternehmensverbände. Diese wurden jeweils beraten und betreut von Patenverbänden der westdeutschen Mitgliedsorganisationen von Gesamtmetall. Zwischen dem Vorstand der IG Metall und Gesamtmetall bestand Einigkeit, dass die Tarifstrukturen der Bundesrepublik auf das Gebiet der DDR übertragen werden. Da diese in der Metall- und Elektroindustrie von regionalen Tarifverträgen geprägt sind, wurde vereinbart, sie wie folgt $\mathrm{zu}$ übertragen: von Schleswig-Holstein nach Mecklenburg-Vorpommern, von Berlin nach Brandenburg, von Niedersachsen nach Sachsen-Anhalt, von Hessen nach Thüringen und von Bayern nach Sachsen. Zur materiellen Angleichung der Tarifstrukturen konnte in zentralen Gesprächen keine Einigung erreicht werden. Sie wurden

Kay Ohl, Funktionsbereichsleiter Tarifpolitik beim Vorstand der IG Metall. e-mail:Kay.Ohl@igmetall.de 
am 28. Juni 1990 abgebrochen. Danach wurde regional über die Einführung der Tarifstrukturen verhandelt.

\section{STUFENPLAN SOLLTE SCHNELLE ANGLEICHUNG BRINGEN}

In den ersten Tarifverträgen, die noch von der IG Metall der DDR geschlossen wurden und denen die IG Metall für die Bundesrepublik Deutschland beitrat, wurden die materiellen Bedingungen zur Einführung der neuen Tarifstrukturen vereinbart. Diese Tarifergebnisse regelten folgende Punkte: Die Verpflichtung, die Tarifstrukturen aus dem jeweiligen Partnergebiet zum 1. April $1991 \mathrm{zu}$ übernehmen; die Absenkung der Arbeitszeit auf 40 Stunden pro Woche; die Vereinbarung eines Jahresgrundurlaubs von mindesten 20 Tagen, eines Kündigungsschutzes bis zum 30. Juni 1991 und einer pauschalen Anhebung der Löhne und Gehälter um 250 DM zum 1. Juli 1990 sowie um weitere 300 DM zum 1. Oktober 1990; die Anerkennung der bisher von der IG Metall der DDR geschlossenen Tarifverträge (mit Ausnahme eines Rationalisierungsschutz-Abkommens).

Nach Inkrafttreten des Einigungsvertrages am 3. Oktober 1990 und nach dem Auflösungsbeschluss der IG Metall der DDR wurden die Tarifverhandlungen über die Übertragung der Tarifstrukturen im Herbst 1990 nun von der für Gesamtdeutschland zuständigen IG Metall geführt.

Am 28. Februar 1991 konnte in Mecklenburg-Vorpommern ein Pilotabschluss erzielt werden. Er sah eine stufenweise Angleichung der tariflich geregelten Arbeitsbedingungen an die Schleswig-Holsteins vor. Danach sollten die Löhne, beginnend mit $62,6 \%$ des Niveaus von SchleswigHolstein, und die Gehälter, beginnend mit 58,6\%, stufenweise bis 1. April 1994 auf $100 \%$ des schleswig-holsteinischen Lohnbzw. Gehaltsniveaus ansteigen. Neben diesem Stufenplan für die Angleichung von Löhnen und Gehältern wurden ebenso die stufenweise Einführung der im Westen tariflich geregelten Leistungszulage, die Anhebung des Urlaubs auf bis zu 30 Tage, die Einführung des Urlaubsgelds und der betrieblichen Sonderzahlung vereinbart. Bei der Arbeitszeit war eine Absenkung in zwei Stufen für den 1. April 1994 auf 39 Stunden pro Woche und am 1. Oktober 1996 auf 38 Stunden pro Woche vereinbart. Die Grundsätze dieses Pilotabschlusses

Tabelle 1: Mitgliederentwicklung in den Verbänden von Gesamtmetall - Neue Bundesländer, Betriebe mit Tarifbindung

\begin{tabular}{lccc}
\hline & Betriebe & Beschäftigte & $\begin{array}{c}\text { Durchschnittliche } \\
\text { Betriebsgröße }\end{array}$ \\
\hline 1990 & 1.192 & 943.827 & 792 \\
1991 & 1.365 & 535.066 & 392 \\
1992 & 1.278 & 270.924 & 212 \\
1993 & 1.111 & 204.458 & 184 \\
1994 & 983 & 163.725 & 167 \\
1995 & 792 & 141.748 & 179 \\
1996 & 655 & 119.718 & 183 \\
1997 & 540 & 100.423 & 186 \\
1998 & 504 & 90.136 & 179 \\
1999 & 442 & 85.256 & 193 \\
2000 & 426 & 84.214 & 198 \\
2001 & 396 & 82.116 & 207 \\
2002 & 353 & 77.612 & 220 \\
2003 & 290 & 67.490 & 233 \\
2004 & 266 & 57.823 & 217 \\
2005 & 240 & 64.622 & 269 \\
2006 & 236 & 65.118 & 276 \\
2007 & 214 & 66.921 & 313 \\
2008 & 212 & 68.318 & 322 \\
\hline
\end{tabular}

Quelle: www.gesamtmetall.de (Stand 31.8.2009); Berechnungen des Autors.

WSI MITTEILUNGEN

wurden auf die übrigen Tarifgebiete in den neuen Ländern übertragen. Der Tarifkompromiss sah die Option vor, nach dem 1. Januar 1993 Verhandlungen über die Durchführung der Vereinbarung aufzunehmen.

Der Stufenplan entsprach der politischen Absicht, die Angleichung der Lebensverhältnisse im Osten Deutschlands an die des Westens möglichst in schnellen Schritten zu vollziehen. Dieses entsprach der politischen Stimmung, die der damalige Bundeskanzler Helmut Kohl mit seinen unrealistischen Aussagen über die blühenden Landschaften geschürt hatte. Für ökonomisch begründete Prognosen und Szenarien einer Ost-West-Angleichung fehlte jede empirische Grundlage. Den Kanzlerkandidaten der SPD, Oskar Lafontaine, straften vor allem ostdeutsche Wähler bei den ersten Wahlen nach der Deutschen Einheit ab für seine realistische Skepsis, dass die Angleichung der wirtschaftlichen Verhältnisse sich nicht so schnell vollziehen würde. Die Tarifvertragsparteien in der Metall- und Elektroindustrie entschieden sich dafür, die Angleichung der tariflichen Einkommens- und Arbeitsbedingungen in einem Stufenplan festzulegen, um damit den Menschen im Osten Deutschlands eine Perspektive der Angleichung ihrer Lebensverhältnisse zu geben. Dies musste in einem überschaubaren Zeitraum geschehen

\section{BETRIEBE WURDEN PRIVATISIERT STATT SANIERT}

Jedoch hatte die Einführung der D-Mark im Osten den abrupten Zusammenbruch der bisher auf die östlichen Staaten ausgerichteten Absatzmärkte zur Folge. Das wiederum führte dazu, dass die Betriebe Kurzarbeit anmelden mussten - dabei wurde im Nachkriegs-Deutschland zum ersten Mal der Begriff „Kurzarbeit Null“ eingeführt. Vor dem Hintergrund des in der Metallund Elektroindustrie bis 30. Juni 1991 vereinbarten Kündigungsschutzes wurde für die Beschäftigten dieser Branche eine Anschlussperspektive gesucht.

Die IG Metall forderte Sanierungsmaßnahmen für die Betriebe und Qualifizierungsmaßnahmen für die Beschäftigten. Die Treuhand orientierte allein darauf, die bisher volkseigenen Betriebe aufzuspalten und an Investoren zu verkaufen, oft zu einem negativen Kaufpreis. Die Betriebe wurden in der Folge häufig nicht saniert, sondern von den meist westdeutschen Investoren geschlossen. Zuvor hatten diese noch bestehende Vermögenswerte verwertet, danach entledigten sie sich ihrer Verantwortung. Die von der IG Metall vorgeschlagene Idee von Beschäftigungsgesellschaften, die Politiker vor Herstellung der Einheit stets abgelehnt hatten, wurde jetzt aufgegriffen. In allen neuen Bundesländern wurden sie gegründet. Sie 


\begin{tabular}{|c|c|c|c|c|c|c|}
\hline & $\begin{array}{l}\text { Entgelte des } \\
\text { Westniveaus } \\
\text { in \% }\end{array}$ & $\begin{array}{l}\text { Leistungs- } \\
\text { zulage } \\
\text { in \% }\end{array}$ & $\begin{array}{l}\text { Arbeitszeit } \\
\text { Std. }\end{array}$ & $\begin{array}{l}\text { Urlaubsdauer } \\
\text { Arbeitstage }\end{array}$ & Urlaubsgeld & $\begin{array}{l}\text { Sonder- } \\
\text { zahlung } \\
\text { in \% }\end{array}$ \\
\hline $\begin{array}{l}1990 \\
\text { 1. Jul. } \\
\text { 1. Okt. }\end{array}$ & $\begin{array}{l}250 \mathrm{DM} \\
\text { pauschal } \\
300 \mathrm{DM} \\
\text { pauschal }\end{array}$ & & 40 & 20 & & \\
\hline $\begin{array}{ll}1991 & \\
1991 & \\
& \text { 1. Apr. }\end{array}$ & $\begin{array}{l}\text { Löhne } 62,6 \\
\text { Gehälter 58,6 }\end{array}$ & Arbeiter 5 & & & & 20 \\
\hline $\begin{array}{l}1992 \text { 1. Apr. } \\
\text { 1. }\end{array}$ & $\begin{array}{c}\text { Löhne } 71 \\
\text { Gehälter } 69\end{array}$ & Arbeiter 7 & & 24 & & 30 \\
\hline $\begin{array}{l}1993 \text { 1.Apr. } \\
\text { 1. Jun. } \\
\text { 1. Sep. } \\
\text { 1. Dez. }\end{array}$ & $\begin{array}{l}75 \\
78 \\
80\end{array}$ & $\begin{array}{c}\text { Arbeiter } 10 \\
\text { Angestellte } 2\end{array}$ & & & & 40 \\
\hline $\begin{array}{ll} & \\
& \text { 1. Apr. } \\
& \text { 1. Jul. }\end{array}$ & 87 & Angestellte 4 & 39 & 27 & & 50 \\
\hline $\begin{array}{l}1995 \\
\text { 1.Apr. } \\
\text { 1. Jul. }\end{array}$ & 94 & $\begin{array}{c}\text { Arbeiter } 16 \\
\text { Angestellte } 6\end{array}$ & & 29 & $\begin{array}{l}50 \% \text { je } \\
\text { Urlaubstag }\end{array}$ & \\
\hline $\begin{array}{ll}1996 & \\
& \text { 1. Jul. } \\
& \text { 1. Okt. }\end{array}$ & 100 & & 38 & 30 & & \\
\hline
\end{tabular}

nahmen Arbeitnehmer auf, die 1991 nach Auslaufen des Kündigungsschutzes arbeitslos wurden.

In den Jahren 1991 bis 1993 änderte sich die Struktur der Mitgliedsunternehmen der Arbeitgeberverbände grundlegend (Tabelle 1). Während die Zahl der Betriebe nach der Statistik von Gesamtmetall nahezu konstant blieb, sank die Zahl der Beschäftigten in den tarifgebundenen Betrieben von $943.000 \mathrm{im}$ Jahr 1990 auf 204.000 im Jahr 1993. Dieser Rückgang drückt den massiven Strukturwandel und Arbeitsverlust in den ersten Jahren der Einheit aus.

\section{ARBEITGEBER VERLANGTEN REVISION DES STUFENPLANS}

Anfang 1993 forderten die Arbeitgeberverbände in der Metall- und Elektroindustrie, unter Berufung auf eine entsprechende Klausel, über eine Revision der bestehenden Stufentarifverträge zu verhandeln. Die Verhandlungen blieben ergebnislos. Die Arbeitgeberverbände leiteten daraufhin ein Schlichtungsverfahren ein, das ebenfalls ohne Ergebnis endete. Danach kündigten die Arbeitgeber zum 31. März 1993 außer-

\section{GESTRECKTER STUFENPLAN UND HÄRTEFALLKLAUSEL}

Der Stufenplan, der bei Löhnen und Gehältern die Angleichung bis 1994 auf $100 \%$ des West-Niveaus vorgesehen hatte, wurde in seiner Laufzeit bis 1. Juli 1996 gestreckt. Eine Besonderheit des Verhandlungsergeb- nisses war die Vereinbarung einer „Härtefallklausel“. Danach sollten vor allem zur Abwendung einer drohenden Insolvenz, zur Sicherung von Arbeitsplätzen, insbesondere zur Vermeidung drohender Entlassungen sowie zur Verbesserung der Sanierungschancen aufgrund eines vorgelegten Sanierungsplanes abweichende Regelungen zwischen den Tarifvertragsparteien geschlossen werden. Über das Vorliegen eines solchen Härtefalls mussten sich die Tarifvertragsparteien verständigen. Kam keine Verständigung zustande, wurde die Schlichtung angerufen. Kam es auch hier zu keinem Ergebnis, galten die Bestimmungen des Flächentarifvertrags. Die Härtefallklausel war in ihrer Laufzeit an den Zeitraum bis zur vollen Angleichung der Löhne und Gehälter gebunden.

Die Einführung der Härtefallklausel markiert eine Zäsur für das Tarifvertragssystem. Der damalige Hauptgeschäftsführer von Gesamtmetall, Dieter Kirchner, feierte die Klausel vor bayerischen Arbeitgebern als betriebswirtschaftlichen Prüfstand, auf den jetzt jeder Tarifvertrag gestellt würde. In Anwendung der Härtefallklausel kam es zu zahlreichen Schlichtungsverfahren zwischen den Tarifvertragsparteien. Hierbei zeigte sich, dass mit der Klausel weniger der Flächentarifvertrag auf dem Prüfstand der Betriebswirtschaft stand, vielmehr wurden die betriebswirtschaftlichen Entscheidungen auf den Prüfstand der Tarifvertragsparteien gestellt. Unternehmen, die von der Härtefallklausel Gebrauch machen wollten, hatten Sanierungspläne vorzulegen. ${ }^{1}$ Nach dieser Öffnung für betriebliche Abweichungen haben die Arbeitgeber die Bestimmungen des gestreckten Stufenplans zur Angleichung der tariflichen Arbeitsbedingungen eingehalten ( $\mathrm{Ta}$ belle 2).

\section{EFFEKTIVVERDIENSTE BLEIBEN UNGLEICH}

Während die tariflichen Grundeinkommen von 1991 bis 1996 von rund $60 \%$ auf $100 \%$ des West-Niveaus stiegen, erhöhten sich die effektiven Einkommen nur von $43,7 \%$ auf $68,8 \%$ bei den Arbeitern und
Vgl. Hickel, R./Kurtzke, W. (1997): Tarifliche Lohnpolitik unter Nutzung der Härtefallregelung: Ergebnisse einer Untersuchung zur Praxis der ostdeutschen Metall- und Elektroindustrie. Zusammenfassung der Studie, Manuskript, Bremen. 
auf 72,8 \% bei den Angestellten (Tabelle 3). Die Statistik der Effektivverdienste weist aus, dass das Entgeltniveau im Ost-WestVergleich seit diesem Zeitraum bei den Arbeitern bei rund $70 \%$ und bei den Angestellten bei rund $75 \%$ verharrt. Eine Angleichung der Effektivverdienste konnte nicht durchgesetzt werden. Das ist vor allem auf zwei Faktoren zurückzuführen.

Während in den westlichen Tarifgebieten häufig übertarifliche Zulagen gezahlt wurden und werden, verharren die Arbeitgeber in den neuen Bundesländern meist auf Tarifniveau. Nur für wenige Beschäftigte, insbesondere im Bereich qualifizierter Angestellter, die vom Westen in den Osten gezogen sind, wurden und werden dort übertarifliche Zulagen gezahlt. Dies führt in vielen Betrieben zu Ungerechtigkeiten: Bei gleicher Qualifikation und gleichen Arbeitsanforderungen erhalten einzelne Beschäftigte nur aufgrund ihrer Übersiedlung in den Osten eine übertarifliche Zulage.

Eine zweite Ursache für die Differenz bei den Effektiveinkommen ist das Fehlen leistungsbezogener Entgeltbestandteile in östlichen Tarifgebieten. Während in westdeutschen Betrieben neben dem Zeitlohn insbesondere in den Produktionsbereichen auch Akkord- und Prämienlöhne angewandt werden, ist dies in den östlichen Bundesländern die Ausnahme. Daher sind die Löhne im Osten Deutschlands auf das Tarifniveau plus der tariflichen Leistungszulage begrenzt. Darüber hinausgehende Akkord- und Prämienverdienste sind für Beschäftigte im Osten nicht erreichbar.

\section{BILLIGER KANN ZUKUNFT KOSTEN}

Die Zahl der Beschäftigten in der Metallund Elektroindustrie sank von $428.000 \mathrm{im}$ Jahr $1992^{2}$ auf $266.000 \mathrm{im}$ Jahr 1997. Seit 1997 steigt die Zahl der Beschäftigten in der ostdeutschen Metall- und Elektroindustrie allerdings kontinuierlich an. Sie erreichte 2008 wieder 422.000. In West-Deutschland hingegen verringerte sie sich von 3,9 Mio. (1992) auf 3,2 Mio. (2008). Diese Entwicklung könnte zu der Annahme verleiten, der Osten Deutschlands hätte den Strukturwandel geschafft. Die Entwicklung der Beschäftigtenzahl drückt jedoch etwas anderes aus. Die Zunahme im Osten Deutschlands ist darauf zurückzuführen, dass westliche Unternehmen Produktionsstätten dorthin verlagert haben. Sie haben sich u. a.

Tabelle 3: Effektivverdienste in der Metall- und Elektroindustrie (Ost/Westvergleich)

\begin{tabular}{lcccccc} 
Jahr & $\begin{array}{c}\text { West } \\
\text { in } €\end{array}$ & $\begin{array}{c}\text { Arbeiter } \\
\text { Ost } \\
\text { in } €\end{array}$ & $\begin{array}{c}\text { Ost/West } \\
\text { in \% }\end{array}$ & $\begin{array}{c}\text { West } \\
\text { in } €\end{array}$ & $\begin{array}{c}\text { Angestellte } \\
\text { Ost } \\
\text { in } €\end{array}$ & $\begin{array}{c}\text { Ost/West } \\
\text { in \% }\end{array}$ \\
\hline 1991 & 11,35 & 4,96 & $\mathbf{4 3 , 7}$ & 2.802 & 1.069 & $\mathbf{3 8 , 2}$ \\
1992 & 11,96 & 6,19 & $\mathbf{5 1 , 8}$ & 2.955 & 1.416 & $\mathbf{4 7 , 9}$ \\
1994 & 13,19 & 8,39 & $\mathbf{6 3 , 6}$ & 3.160 & 2.025 & $\mathbf{6 4 , 1}$ \\
1996 & 14,27 & 9,82 & $\mathbf{6 8 , 8}$ & 3.415 & 2.485 & $\mathbf{7 2 , 8}$ \\
1998 & 14,88 & 10,53 & $\mathbf{7 0 , 8}$ & 3.553 & 2.684 & $\mathbf{7 5 , 5}$ \\
2000 & 15,65 & 10,78 & $\mathbf{6 8 , 9}$ & 3.687 & 2.769 & $\mathbf{7 5 , 1}$ \\
2002 & 16,12 & 11,32 & $\mathbf{7 0 , 2}$ & 3.910 & 2.945 & $\mathbf{7 5 , 3}$ \\
2004 & 16,99 & 11,76 & $\mathbf{6 9 , 2}$ & 4.102 & 3.061 & $\mathbf{7 4 , 6}$ \\
2006 & 17,38 & 12,00 & $\mathbf{6 9 , 0}$ & 4.245 & 3.137 & $\mathbf{7 3 , 9}$ \\
\hline \multicolumn{7}{r}{} \\
Quelle: www.gesamtmetall.de; Statistisches Bundesamt.
\end{tabular}

die Lohnkostenvorteile im Osten zunutze gemacht. $\mathrm{Zu}$ bezweifeln ist, ob die neuen Bundesländer mit einer derartigen Billigstrategie Zukunft haben.

Das Niveau der Löhne in der ostdeutschen gegenüber der westdeutschen Industrie ist ständig gesunken und lag 2008 bei $72 \%$. Die Produktivität hingegen - gemessen als Produktion je Beschäftigtenstundeist stetig gestiegen und lag 2008 bei 78,3\% des West-Niveaus. Diese Entwicklung hat sinkende Lohnstückkosten zur Folge, was sich als Lohnkostenvorteil Ost auswirkt. Angesichts dieser Entwicklung von Lohnund Produktivitätsniveau besteht für eine weitere Angleichung der Effektiventgelte ausreichender wirtschaftlicher Spielraum.

\section{WENIGER BINDEKRAFT, MEHR ORIENTIERUNGSFUNKTION}

Die Angleichung der tariflich geregelten Arbeitsbedingungen begann im Jahr 1990 mit dem großen gesellschaftlichen Konsens, gleichwertige Lebensverhältnisse in allen Regionen Deutschlands herzustellen. Diesen Konsens haben die Arbeitgeber 1993 mit der Kündigung der Stufentarifverträge aufgekündigt.

Die Entwicklung der Tarifbindung im Osten Deutschlands zeigt, dass sich die in der Bundesrepublik bewährte Tarif- und Mitbestimmungskultur in den östlichen Bundesländern nicht im gleichen Maße durchgesetzt hat. Insbesondere kleine und mittelständische Unternehmen, deren Zahl deutlich gestiegen ist, bleiben den Verbänden der Metall- und Elektroindustrie fern. Allerdings lehnen sich diese Unternehmen bei der Gestaltung der betrieblichen Arbeitsbedingungen häufig an die Flächen- tarifverträge an, sodass ,in einem umfangreichen Maßstab Regelungen des Branchentarifvertrages in nicht arbeitgeberverbandsgebundenen Unternehmen angewandt werden ". ${ }^{3}$ Laut IAB-Betriebspanel ${ }^{4}$ hatten sich z. B. in Thüringen $200353 \%$ der Betriebe des verarbeitenden Gewerbes, die $74 \%$ aller Beschäftigten repräsentieren, den tariflichen Regelungen angeschlossen oder orientierten sich daran.

Im gleichen Jahr unternahm die IG Metall den Versuch, auch die Arbeitszeit an das westliche Niveau von 35 Stunden/Woche anzugleichen. Dieser Versuch ist gescheitert. Jedoch spätestens mit dem breiten Einsatz des Instruments Kurzarbeit in der Wirtschaftskrise 2009 kann die Debatte um Arbeitszeitverkürzung zur Beschäftigungssicherung ohne Ideologie geführt werden. Verkürzung ist ein geeignetes Instrument zur Verteilung eines begrenzten Arbeitvolumens. Kürzere Arbeitszeiten schließen Wachstum und Wohlstand nicht aus. Die Ost-West-Angleichung der tariflichen Arbeitsbedingungen ist für die Metall- und Elektroindustrie auch fast 20 Jahre nach der deutschen Vereinigung nicht abgeschlossen.

21990 und 1991 waren viele der Beschäftigten in Kurzarbeit Null noch Angehörige des Betriebes, weshalb keine verlässliche Aussage über die tatsächliche Anzahl der "produktiv“ Beschäftigten gemacht werden kann.

3 Finley, P./Weitz, R. (2004): Firmen-, anerkennungs- und unternehmensbezogene Verbandstarifverträge im Spiegel flächentariflicher Regelungen in der Metall- und Elektroindustrie Thüringens, Erfurt.

4 IAB-Betriebspanel (2003): Länderbericht Thüringen. 